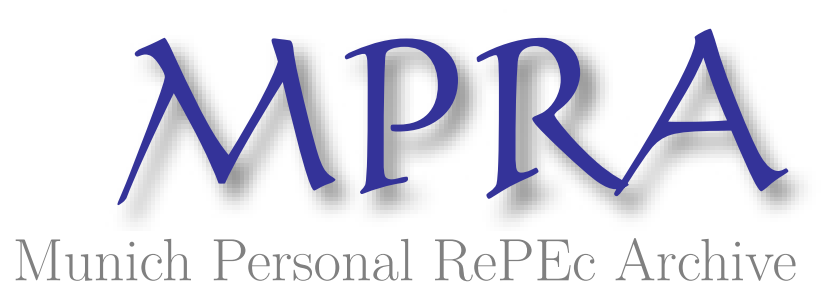

\title{
The Service Quality Undergraduate - A Case of Public Education
}

Onusic, Luciana

Unip

28 April 2011

Online at https://mpra.ub.uni-muenchen.de/34102/

MPRA Paper No. 34102, posted 15 Oct 2011 10:11 UTC 


\title{
The Service Quality Undergraduate - A Case of Public Education
}

\author{
Luciana Massaro Onusic \\ Assistant Professor at Universidade Paulista-Unip \\ São Paulo - Brazil \\ lucianaonusic@gmail.com
}

\begin{abstract}
A growing number of courses have been created lately in higher education in Brazil. The quality of these courses must be maintained to ensure that professionals are prepared for the labour market. The present work aims to identify, through a case study, the factors that contribute so that the student does not abandon the course s/he chose in a public higher education institution, FEA/USP. The students who participated in this study are those who started the courses in Management, Accountancy, Actuarial Science and Economics in 2007. In order to develop this work, some steps were followed: review of relevant literature and development of a model used in the research. Then a quantitative study was structured to conducted with the students from FEA/USP, and finally, a quantitative analysis was performed using quantitative analysis techniques in order to examine the results obtained. The model chosen to frame this study was the one proposed by Bloemer (1998), which was previously applied to financial institutions. In the present study, the model was used in a higher education institution with constructs of quality, image, satisfaction and loyalty. To assess the relationship among the above-mentioned constructs, structural equations technique were used. As a result of the analysis, it could be observed that student's loyalty comes directly from his or her general satisfaction with the course, which is influenced by the course's perceived quality. It is important to observe that the aspects that most distinguish loyal students from non-loyal ones are satisfaction with professors, administrative processes and with administration in general.
\end{abstract}

Key Words: Higher education, Varied Analysis, Loyalty and Quality

JEL Code: I21; I23.

São Paulo, October 2011 


\section{INTRODUÇÃO}

Segundo Fitzsimmons, J. e Fitzsimmons, M. (2005), o setor de educação pode ser considerado um serviço de aperfeiçoamento. Assim, uma Instituição de Ensino Superior, seja ela pública ou privada, constitui uma organização que presta serviços a seus clientes.

Para Martins (2000), o Ensino Superior no Brasil passou por várias mudanças. Existiam pouco mais de cem instituições de ensino superior no início dos anos 60, voltadas, em sua maioria, para a transmissão do conhecimento, com um corpo docente pouco profissionalizado. Segundo o autor, no início da década de 60 , pouco mais de 100 mil alunos matricularam-se nas Instituições de Ensino Superior; número que chegou a mais de 2,1 milhões em 1998.

Dados de 2004 do INEP indicavam que existiam 1859 instituições de ensino superior, sendo divididas em universidades, centros universitários, faculdades integradas, faculdades isoladas, escolas, institutos e centros de educação tecnológica, sendo que 1652 eram privadas (MOISES FILHO, 2006).

Nota-se no estudo de Santos e Noronha (2001), que a qualidade percebida do curso pode ser um fator de desistência do mesmo quando ele não satisfaz o aluno e, no que tange a FEA/USP, o estudo de Graça (1997) mostrou que a avaliação de aspectos tangíveis e corpo docente ficaram aquém do desejado.

Neste contexto, este trabalho propõe-se a investigar a qualidade em serviços educacionais, focando os alunos de ensino superior público, a FEA/USP. Será realizada uma pesquisa quantitativa junto aos alunos desta instituição para posterior mapeamento de suas percepções em relação à qualidade do serviço prestado ao aluno de graduação, bem como sua satisfação em relação ao curso escolhido.

Neste contexto, este trabalho busca responder à seguinte situação-problema: "Quais são os fatores que contribuem para a lealdade do aluno para que este permaneça e termine o curso superior escolhido?"

Este estudo se justifica, pois o setor de educação superior no Brasil, considerado uma prestação de serviços, tem crescido muito nas últimas décadas. A demanda por cursos superiores tanto em Universidades Privadas como em Universidades Públicas tem tornado este mercado competitivo devido à alta exigência de seu público-alvo em diversos itens, sendo um deles a qualidade do serviço oferecido. A falta de qualidade, ou a qualidade percebida como insatisfatória do serviço de educação pode gerar a desistência ou desmotivação em relação aos cursos de graduação.

Neste cenário, o entendimento das percepções dos consumidores, no caso os alunos, surge como um elemento importante para a continuidade destas Instituições.

Assim, este estudo tem sua importância em descobrir os fatores que motivam os alunos a permanecerem na instituição que escolheram, permitindo a busca de soluções a curto e longo prazo para o problema de insatisfação e desistência dos cursos pelos alunos.

\section{REVISÃO DA LITERATURA}

\subsection{O Ensino Superior no Brasil}

O ensino superior no Brasil surgiu sob a forma de faculdades isoladas, ainda no período colonial. Segundo a publicação da FAPESP, Indicadores de Ciência, Tecnologia e Inovação em São Paulo, existiam duas universidades ao final da Primeira República, a universidade do Rio de Janeiro, criada em 1920 pelo Governo Federal e a universidade de Minas Gerais, criada em 1927 pelo Governo Estadual.

Para Moises Filho (2006), a evolução histórica do ensino superior no Brasil possui quatro fases: antes de 1930; entre 1930 e 1964; de 1964 a 1980 e de 1980 a 1996.

$>$ Antes de 1930: predomínio das instituições públicas

> 1930 a 1964: ocorre uma consolidação do sistema de ensino privado. 
$>1964$ a 1980: reforma do ensino superior e predominância do setor privado.

$>1980$ a 2002: aumento da oferta de vagas do setor privado, crescimento do número de vagas não preenchidas e evasão.

Chegando ao novo milênio, verifica-se um aumento na oferta de cursos superiores. Das 1859 IES existentes em 2003, 221 eram públicas. Em 2008, este número aumentou para um total de 2270 IES, sendo 248 classificadas como públicas. Em 2008, existiam 183 Universidades, 126 Centros Universitários, 116 Faculdades Integradas, 1649 Faculdades, Escolas e Institutos e 208 Centros Tecnológicos (INEP, 2008).

A tendência de aumento da oferta de vagas pelas IES continua até os dias de hoje, mostrando um aquecimento do mercado neste tipo de prestação de serviço. No entanto, mesmo com o controle maior da inflação, alguns autores identificam desistências em continuar os estudos por parte de alguns alunos que estão no curso superior.

No período de 1995 a 2002 ocorreram algumas alterações na legislação. Em 1995 foi editada A Lei 9.131, em 24 de novembro, criando-se o Exame Nacional de Curso. Segundo Dias Sobrinho (2003), o Exame Nacional de Curso tem um papel imprescindível na fiscalização das instituições. Para o autor, alguns acadêmicos mais comprometidos com o sistema de ensino vêem com desconfiança o Provão que seria apenas um elemento da privatização e só interessa às forças de mercado por estabelecer esquemas que hierarquizam as instituições de ensino.

\subsection{Marketing para Instituições de Ensino Superior}

No cenário atual, as Instituições de Ensino Superior (IES) estão buscando padrões de excelência para poderem concorrer no mercado cada vez mais competitivo nesta área. Para isto, as Instituições de Ensino estão se profissionalizando, isto é, buscam satisfazer tanto a formação pedagógica do aluno e a preocupação com o ensino-aprendizagem, como também exigir um nível de profissionalização mais avançado por parte de seus dirigentes e corpo técnico-administrativo. É necessária a excelência na forma de gestão para aumentar a competitividade e a sobrevivência das instituições (PERFEITO, 2007). Assim, muitas IES buscam conhecer as expectativas do mercado cliente, pois estão vislumbrando novos horizontes, impostos pela complexidade do ambiente.

Manes (1997, p. 45 apud PERFEITO, 2007, p. 23) apresenta sua definição de Marketing Educacional como sendo: "O processo de investigação das necessidades sociais de aprendizagem, de modo a orientar e desenvolver programas educativos que as satisfaçam".

Neste contexto, se o foco do marketing é o cliente, deve-se conhecer este para poder determinar ações, dado o comportamento destes na pré-compra e pós-compra do produto.

Segundo Kotler \& Fox (1994), as instituições educacionais que estão enfrentando declínio de matrículas, aumento de custos, e que percebem que dependem do mercado para serem mais bem sucedidas, estão recorrendo ao marketing.

Observando-se o contexto de educação no Brasil e sua evolução e abordando-se os motivos de evasão do ensino superior em várias instituições, nota-se que muitos fatores podem ser as causas da desistência do curso escolhido. Problemas com a qualidade do ensino oferecido, tanto de aspectos tangíveis como de corpo docente, podem contribuir para que o aluno opte por desistir do curso que está realizando.

Neste contexto, o marketing voltado às instituições de ensino superior busca conhecer as expectativas do mercado, tentando entender o ambiente em que os clientes (possíveis alunos) estão inseridos para que a instituição possa adequar-se a essa demanda no que tange aos padrões de ensino-aprendizagem, bem como a área técnico/administrativa. 


\subsection{A Relação entre Qualidade, Imagem, Satisfação e Lealdade}

Segundo Masano (2006), foi realizada uma série de estudos para se buscar compreender as relações entre qualidade, imagem, satisfação e lealdade. De acordo com Olsen (2002), para se conquistar clientes com comportamento de lealdade, deve-se pensar na cadeia Desempenho Satisfação - Lealdade, em "que o ponto de intervenção dos gestores de marketing é a qualidade da oferta", ou seja, a percepção de qualidade fornece uma condição para a satisfação, desencadeando a condição de lealdade pelo consumidor.

Faz-se importante citar algumas referências que mostram a relação entre a qualidade percebida e a satisfação do cliente. Selnes (1993, p.48) cita alguns estudos que mostram a relação positiva entre a qualidade percebida e a satisfação do consumidor "Fornell (1992) descobriu em uma pesquisa com consumidores suecos uma correlação entre qualidade percebida e satisfação que varia entre 0,43 (companhias de petróleo) a 0,79 (seguros de propriedade)".

A imagem é um fator interveniente na formação da qualidade percebida pelo consumidor, atuando paralelamente na construção de suas expectativas e percepções. (GRÖNROOS, 1993; ZEITHAML et al., 1990; BLOEMER et al., 1998).

Quanto ao conceito de lealdade, para Prado (2004), este pode ser entendido como a compra repetida. Para outros autores, a lealdade está relacionada com a compra repetida correlacionada com uma atitude relativa favorável para com a empresa (DICK e BASU, 1994).

Um estudo muito interessante que tratou dos quatro conceitos (qualidade, imagem, lealdade e satisfação) foi apresentado por Bloemer (1998), que criou um modelo baseado em análise multivariada, mostrando a relação entre imagem, qualidade, satisfação e lealdade para uma instituição financeira. A Figura 1 apresenta a relação entre esses conceitos.

\section{Figura 1 - Relação entre Imagem, Qualidade, Satisfação e Lealdade}

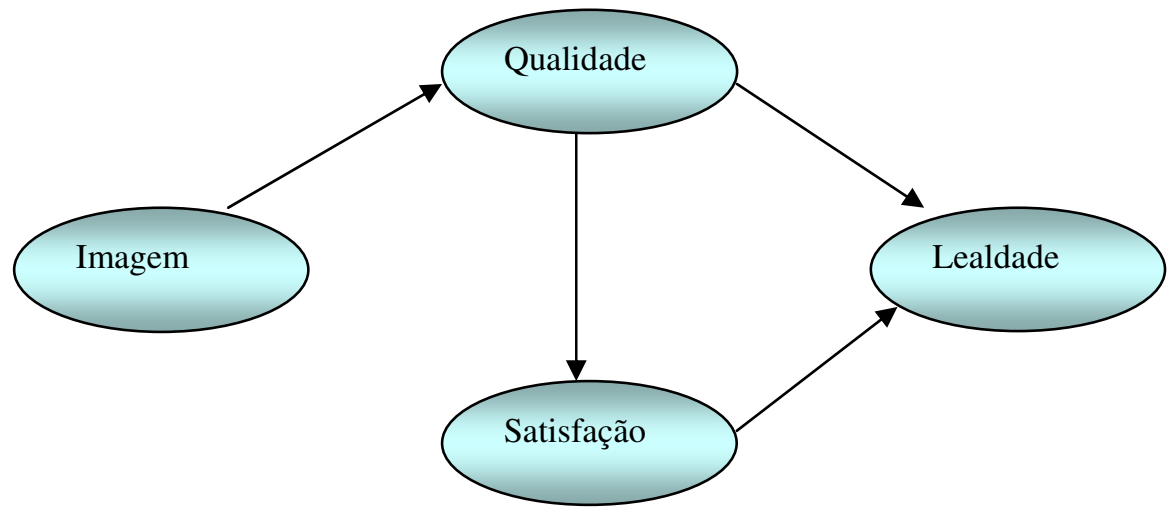

Fonte: Adaptado de Bloemer (1998)

Bloemer (1998) em sua pesquisa pode notar que os três constructos qualidade, imagem e satisfação exercem influência na lealdade do consumidor de instituições financeiras. $\mathrm{O}$ artigo trata primeiramente da construção do conceito de lealdade; conceitua a diferença entre serviço de qualidade e satisfação como determinantes da lealdade. Em seguida, introduz o constructo da imagem, formulando uma hipótese em que a lealdade é resultado dos outros três elementos: qualidade, satisfação e imagem. Para Bloemer (1998), manter a lealdade em serviços é mais dependente de relação interpessoal que em outros tipos de produtos, como na indústria, por exemplo, pois no serviço existe a presença do intangível. 


\section{FUNDAMENTAÇÃO E HIPÓTESES DA PESQUISA}

As hipóteses deste trabalho foram elaboradas tendo como base o modelo de Bloemer (1998) proposto para verificar a relação entre os constructos imagem, qualidade, satisfação e lealdade. Entretanto, uma vez que o modelo de Bloemer (1998) foi proposto para instituições financeiras, algumas adaptações foram realizadas para o caso específico do estudo em questão; assim, o modelo de pesquisa proposto focaliza os constructos de imagem, qualidade, satisfação e lealdade para o setor de ensino superior.

O modelo de Bloemer (1998) testou algumas hipóteses. As mesmas foram adaptadas no estudo em questão. Portanto, o desenvolvimento deste estudo baseia-se fundamentalmente nas seguintes hipóteses:

\section{H1: a qualidade é diretamente influenciada pela imagem. \\ H2: a satisfação é diretamente influenciada pela qualidade. \\ H3: a lealdade é diretamente influenciada pela qualidade. \\ H4: a lealdade é diretamente influenciada pela satisfação.}

Em consonância com o objetivo geral deste estudo, a lealdade dos alunos para sua permanência no curso escolhido será explicada por meio de três constructos: (1) imagem, (2) qualidade, (3) satisfação, conforme modelo de Bloemer (1998) apresentado na ilustração 1.

\section{METODOLOGIA}

De acordo com os objetivos deste estudo foram definidos os tipos de pesquisa, a população e amostragem, o instrumento de coleta de dados e as técnicas estatísticas de análise dos dados coletados.

\subsubsection{Estudo de Caso}

O resultado deste trabalho está pautado nos dados obtidos dos alunos da A FEA (Faculdade de Economia, Administração, Contabilidade e Atuária). Esta Instituição foi criada em 1946, com a denominação de FCEA (Faculdade de Ciências Econômicas e Administrativas) ligada à USP. A FEA é uma instituição pública de ensino e pesquisa e, atualmente, conta com cursos de graduação e pós-graduação em várias áreas, sendo que os cursos de graduação são: Administração, Economia, Ciências Contábeis, Atuária e Relações Internacionais.

\subsubsection{População, Coleta de Dados e Amostra}

A população deste estudo consiste dos alunos ingressantes em 2007 na FEA/USP São Paulo nos cursos de graduação em Administração, Economia, Ciências Contábeis e Ciências Atuariais, nos períodos diurno e noturno, que estão matriculados.

A pesquisa é de natureza quantitativa, descritiva e possibilitou a realização de testes de hipótese e a resposta às indagações que motivaram o presente estudo.

A Coleta de Dados foi realizada com base em questionário estruturado e os resultados foram analisados com o emprego de técnicas estatísticas no contexto uni, bi e multivariado. Desta forma, a pesquisa quantitativa teve como objetivo identificar, com consistência estatística, as percepções dos alunos da FEA-USP sobre qualidade, imagem, satisfação e lealdade à instituição de ensino em que estudam.

Dos alunos que estão devidamente matriculados nos cursos mencionados foram encontrados 600 alunos ingressantes no ano de 2007. Para estes, a coleta de dados foi realizada com o envio de questionário eletrônico.

A amostra final obtida foi de 226 respondentes. O endereço eletrônico do questionário foi enviado via e-mail aos alunos de graduação da FEA/USP. O e-mail com o link para o 
questionário da pesquisa foi enviado durante o mês de dezembro de 2008 e janeiro de 2009 . Foram consideradas para este estudo as respostas recebidas até 03 de março de 2009.

A Figura 2, apresenta a estrutura do questionário aplicado com as respectivas variáveis.

Figura 2 - Constructos e Variáveis

\begin{tabular}{|c|c|c|}
\hline Constructo & Variáveis & $\begin{array}{c}\text { Código } \\
\text { da } \\
\text { variável }\end{array}$ \\
\hline \multirow{35}{*}{ Qualidade } & \multicolumn{2}{|l|}{ Avaliação do corpo docente } \\
\hline & 1. Metodologia de ensino. & V1 \\
\hline & $\begin{array}{l}\text { 2. Conhecimento sobre a realidade dos alunos, facilitando o processo de } \\
\text { aprendizagem. }\end{array}$ & $\mathrm{V} 2$ \\
\hline & 3. Atenção dada às solicitações e intervenções dos alunos. & V3 \\
\hline & 4. Relacionamento com os alunos. & $\mathrm{V} 4$ \\
\hline & 5. Estímulo à participação dos alunos em sala de aula. & $\mathrm{V5}$ \\
\hline & 6. Competência em relacionar teoria com a prática. & V6 \\
\hline & 7. Capacidade para utilizar adequadamente o tempo da aula. & V7 \\
\hline & \multicolumn{2}{|l|}{ Avaliação do corpo técnico-administrativo } \\
\hline & 8. Capacidade para cumprir exatamente as solicitações dos alunos. & V8 \\
\hline & 9. Boa vontade em resolver os problemas acadêmicos dos alunos. & V9 \\
\hline & 10. Agilidade no atendimento. & $\mathrm{V} 10$ \\
\hline & 11. Educação no tratamento com os alunos & V11 \\
\hline & 12. Conhecimento suficiente para fornecer informações aos alunos. & $\mathrm{V} 12$ \\
\hline & \multicolumn{2}{|l|}{ Relacionamento - Discente } \\
\hline & 13. Assiduidade nas aulas do curso. & V13 \\
\hline & 14. Colaboração para manter a limpeza e a conservação da faculdade. & V14 \\
\hline & 15. Respeito entre os alunos. & V15 \\
\hline & 16. Interesse e atenção durante as aulas. & V16 \\
\hline & $\begin{array}{l}\text { 17. Interesse na execução dos exercícios e trabalhos solicitados pelos } \\
\text { professores. }\end{array}$ & V17 \\
\hline & \multicolumn{2}{|l|}{ Avaliação dos aspectos tangíveis } \\
\hline & 18. Iluminação nas dependências do curso. & V18 \\
\hline & 19. Limpeza de todas as dependências da faculdade. & V19 \\
\hline & 20. Alimentação oferecida pela lanchonete. & $\mathrm{V} 20$ \\
\hline & 21. Localização da faculdade. & V21 \\
\hline & 22. Facilidade de navegação e atualização do site. & $\mathrm{V} 22$ \\
\hline & 23. Acervo da biblioteca. & V23 \\
\hline & 24. Equipamentos audiovisuais das salas. & $\mathrm{V} 24$ \\
\hline & 25. Laboratórios e equipamentos de informática. & V25 \\
\hline & 26. Segurança nas dependências da faculdade. & V26 \\
\hline & \multicolumn{2}{|l|}{ Avaliação dos processos administrativos } \\
\hline & $\begin{array}{l}\text { 27. Facilidade na obtenção de informações sobre as regras e normas da } \\
\text { faculdade. }\end{array}$ & V27 \\
\hline & 28. Rapidez e facilidade no processo de matrícula e retificação. & V28 \\
\hline & $\begin{array}{l}\text { 29. Facilidade na obtenção do histórico escolar, carteirinhas, aproveitamento de } \\
\text { estudos. }\end{array}$ & V29 \\
\hline & 30. Adequação da carga horária do curso. & $\mathrm{V} 30$ \\
\hline \multirow{4}{*}{ Satisfação } & 31. Satisfação em estudar na FEA. & V31 \\
\hline & 32. Satisfação com a FEA em relação a uma faculdade ideal. & V32 \\
\hline & 33. Grau em que a FEA atende as suas expectativas. & V33 \\
\hline & 34. Adequada abrangência curricular. & V34 \\
\hline \multirow{5}{*}{ Imagem } & 35. Instituição ética. & $\mathrm{V} 35$ \\
\hline & 36. Tem tradição. & V36 \\
\hline & 37. Socialmente responsável. & V37 \\
\hline & 38. Inovadora. & $\mathrm{V} 38$ \\
\hline & 39. Avançada tecnologicamente. & V39 \\
\hline
\end{tabular}




\begin{tabular}{l|l|c}
\hline & 40. Dá prestígio aos alunos. & V40 \\
\hline \multirow{4}{*}{ Lealdade } & 41. Mencionar a outras pessoas aspectos positivos da FEA. & V41 \\
\cline { 2 - 3 } & 42. Recomendar a FEA a parentes e amigos. & V42 \\
\cline { 2 - 3 } & 43. Considera a FEA como a primeira opção caso fosse fazer outro curso. & V43 \\
\cline { 2 - 3 } & 44. Pretende continuar no curso até se formar. & V44 \\
\cline { 2 - 3 } & 45. Tem orgulho de ser aluno(a) da FEA. & V45 \\
\hline \multirow{4}{*}{$\begin{array}{c}\text { Características amostra } \\
\text { da am }\end{array}$} & 46. Curso & V46 \\
\cline { 2 - 3 } & 47. Ano de ingresso no curso & V47 \\
\cline { 2 - 3 } & 48. Sexo & V48 \\
\cline { 2 - 3 } & 49. Idade & V49 \\
\cline { 2 - 3 } & 50. Período em que estuda & V50 \\
\hline
\end{tabular}

Fonte: elaborado pelo autor

\section{PROCEDIMENTOS PARA ANÁLISE DOS RESULTADOS}

Será apresentado a seguir os procedimentos para análise dos dados da pesquisa.

\subsection{Tratamento prévio dos dados}

Antes da aplicação das técnicas de análise de dados realizou-se um exame prévio dos dados envolvendo toda a amostra. Examinou-se a freqüência de cada variável, a normalidade, a existência das respostas não dadas (missing) e de observações atípicas (outliers). Foram calculadas as principais estatísticas descritivas. $\mathrm{O}$ tratamento prévio dos dados realizou-se levando em consideração as limitações impostas pelos tipos de escalas de cada variável.

Para o processamento dos dados deste capítulo foi utilizada a ferramenta (software) de análise multivariada SPSS - Statistical Package for Social Sciences, versão 9.0 e SmartPLS 2.0 M3 (RINGLE, WENDE e WILL, 2005).

\subsection{Modelagem de Equações Estruturais}

Para identificar as relações existentes entre os conceitos de imagem, qualidade e satisfação dos alunos e o grau de lealdade destes em relação ao curso escolhido na FEA/USP, foi utilizada no contexto dos atributos da qualidade a escala SERVPERF (modelo baseado na percepção de desempenho dos serviços, segundo Cronin e Taylor (1992) ) e modelo de Bloemer (1998), adaptado ao contexto de ensino superior.

Para Klem (1995) apud Farias e Santos (2000, p.113), a modelagem de equações estruturais (MEE ou Structural Equations Modeling - SEM) pode ser vista como a extensão da regressão múltipla.

Para Niño (2009, p. 114)

A modelagem de equações estruturais é uma técnica de análise multivariada que combina elementos de regressão múltipla (examinando relações de dependência) e da análise fatorial (representando fatores ou constructos não diretamente observados), para estimar uma série de relações de dependência simultaneamente. De modo geral, Hair JR et al. (2005a, p.470) distinguem todas as técnicas de modelagem de equações estruturais por duas características: “(i) estimação de múltiplas e inter-relacionadas relações de dependência e (ii) a habilidade para representar conceitos não observados nessas relações e explicar erros de mensuração no processo de estimação.

Para a estimação dos modelos de mensuração e estrutural, a MEE apresenta alguns métodos, sendo os mais conhecidos o baseado em covariâncias (BC) e o Partial Least Squares (PLS). Para este estudo foram utilizadas as seguintes medidas de qualidade do modelo:

AVE: Average Variance Explained ou variância média explicada calculada por constructo ou variável latente.

$\sim \mathbf{R}^{2}$ : variância explicada; grau em que as variáveis dependentes são previstas pelas independentes. 
Goodness-of-fit (Gof): Índice de adequação do modelo que mede quanto da variância é explicada pelo modelo, proposto por Tenenhaus et al (2005) apud ZWICKER, SOUZA e BIDO (2008, p.5). Basicamente é a média geométrica entre o $\mathrm{R}^{2}$ médio (adequação do modelo estrutural) e a AVE média ponderada pela quantidade de indicadores (adequação do modelo de mensuração). Esta medida é aplicável a modelos em que todas as variáveis latentes ou indicadores são reflexivos.

\subsection{Análises multivariadas}

A seguir, apresentam-se os principais resultados obtidos a partir da aplicação das técnicas de análise multivariada; no entanto, é necessária a análise do modelo proposto antes da aplicação das técnicas.

\subsubsection{Análise do modelo de pesquisa proposto}

A análise do modelo de pesquisa proposto foi realizada mediante avaliação do modelo estrutural e análise de testes paramétricos e não-paramétricos para investigação da influência das variáveis moderadoras: sexo, curso, período do curso nos constructos: imagem, qualidade, satisfação e lealdade, conforme Figura 3.

\begin{tabular}{c|c}
\begin{tabular}{c} 
Figura 3- Resumo das hipóteses \\
\hline $\begin{array}{c}\text { Número } \\
\text { da } \\
\text { Hipótese }\end{array}$
\end{tabular} \\
\hline $\mathrm{H}_{1}$ & A qualidade é influenciada pela imagem da instituição \\
\hline $\mathrm{H}_{2}$ & A satisfação é influenciada pela qualidade percebida \\
\hline $\mathrm{H}_{3}$ & A lealdade é influenciada pela qualidade \\
\hline $\mathrm{H}_{4}$ & A lealdade é influenciada pela satisfação \\
\hline Fonte: elaborado pelo autor
\end{tabular}

\subsubsection{Avaliação do modelo estrutural}

Com os resultados do modelo de mensuração (Modelo Final Ajustado), procedeu-se para avaliação do modelo estrutural, estimado por meio do bootstrapping com N=226 e 2000 repetições. Mostram-se significantes ao nível de 5\%, as relações da imagem com a qualidade, a qualidade com a satisfação e esta com a lealdade. Na Ilustração 20, apresentam-se os coeficientes estruturais dos constructos com destaque em negrito para os que apresentam significância no teste t de Student. Os indicadores e respectivas cargas foram omitidos para simplificação. No modelo da Erro! Fonte de referência não encontrada., o Good-of-fit foi igual a 0,5319 e esse valor poder ser interpretado como se fosse um $\mathrm{R}^{2}$ geral, que leva em conta a adequação do modelo de mensuração e do modelo estrutural. Tenenhuaus et al (2005) apud Bido et al (2008, p.14) não propõem um ponto de corte desta medida, entretanto, é possível aceitar este resultado como adequado, já que o critério mínimo para o valor da AVE foi próximo de 0,5 . 
Figura 4 - Modelo de mensuração e estrutural 1

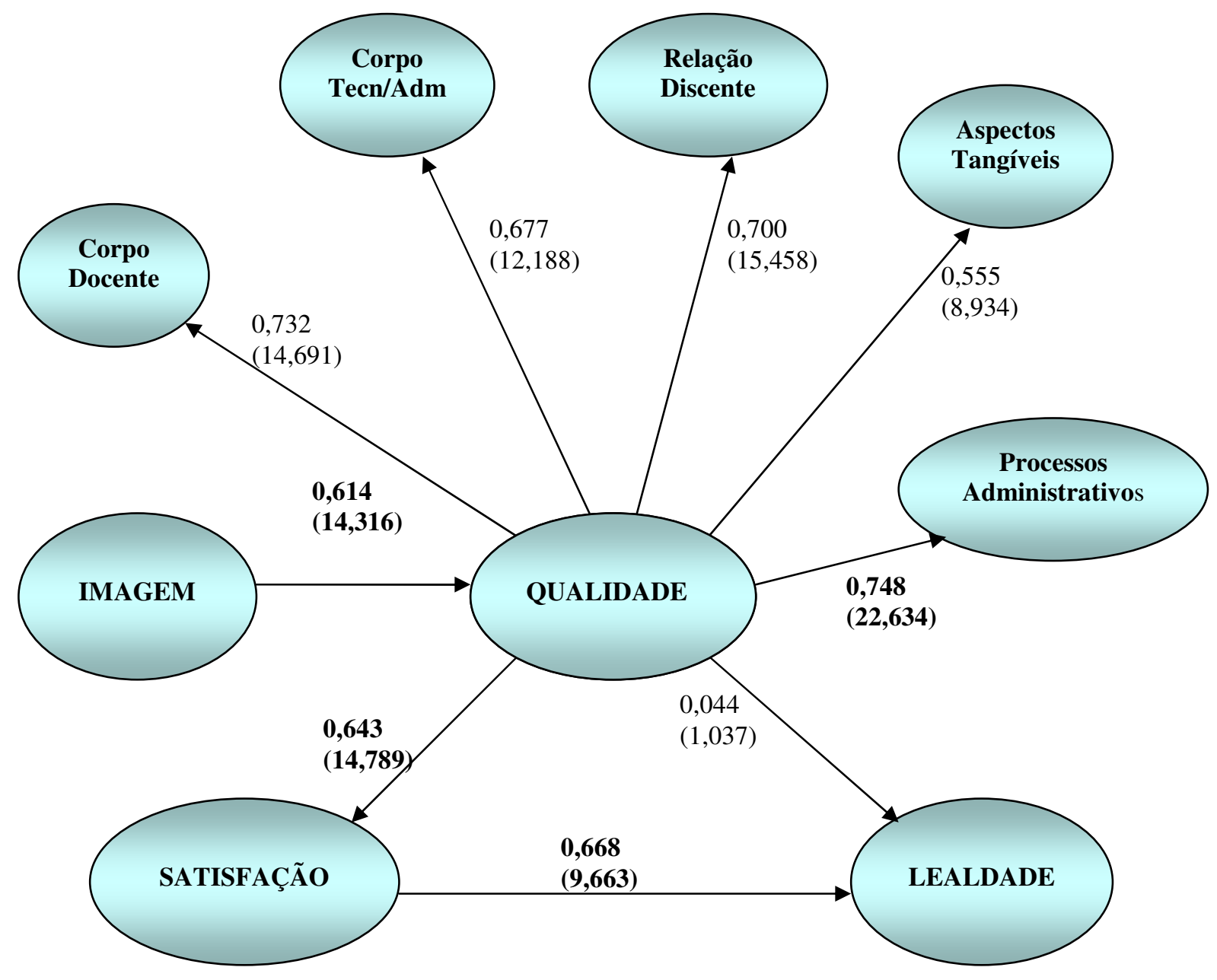

Fonte: Dados coletados e processados.

Posteriormente, aplicou-se novamente o algoritmo PLS, mantendo-se apenas os constructos com efeitos significantes. Figura 5, pode-se visualizar o modelo final ajustado. 
Figura 5 - Modelo final ajustado

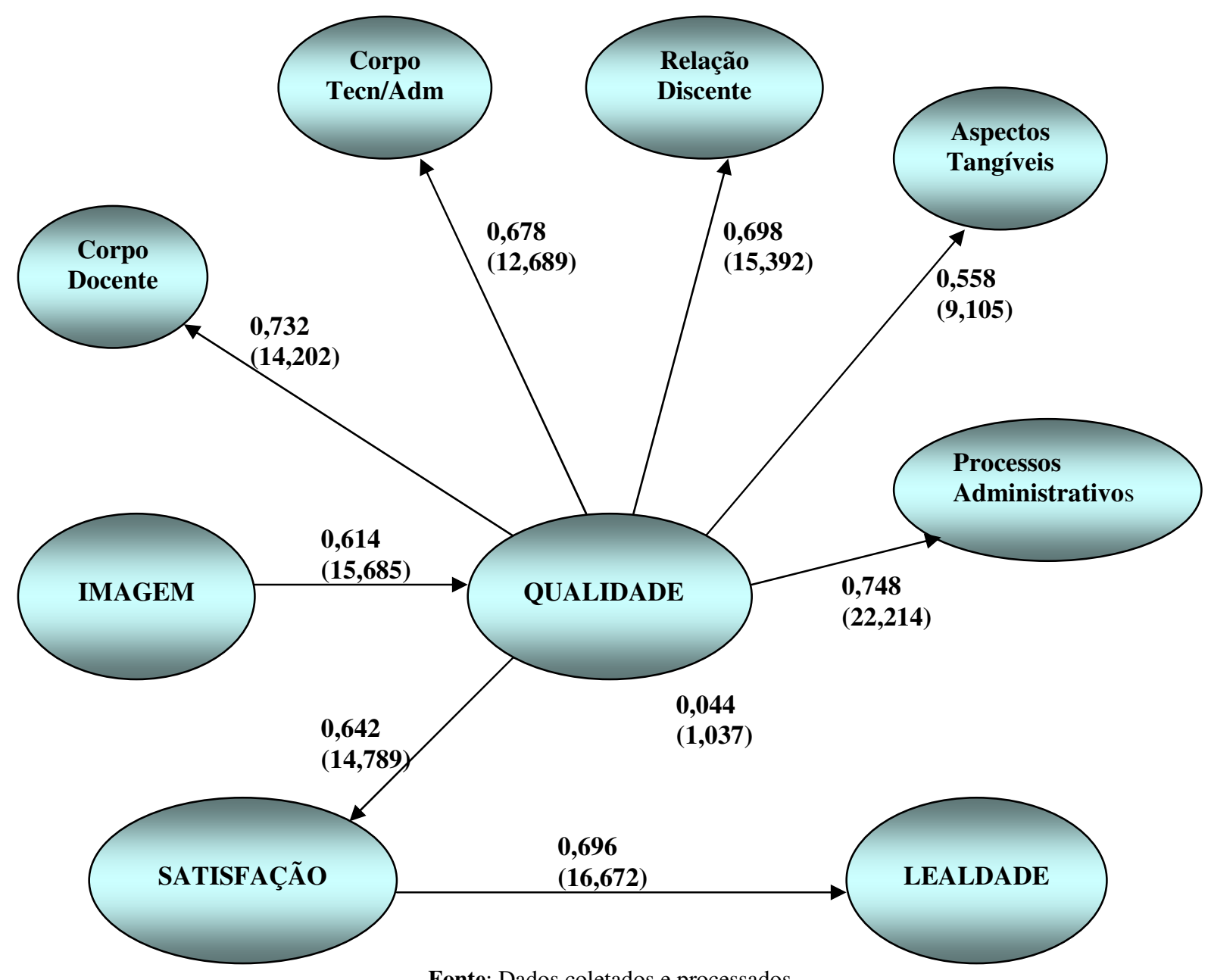

Sob a ótica da análise de caminhos, os efeitos diretos podem ser avaliados, observando-se os valores dos coeficientes estruturais na Tabela 1.

Tabela 1 - Modelo - Ajustado: análise de caminhos

\begin{tabular}{llcclcrr}
\hline \multicolumn{1}{c}{ De } & \multicolumn{1}{c}{ Para } & $\begin{array}{c}\text { Efeito direto } \\
\text { (a) }\end{array}$ & $\begin{array}{c}\text { Efeito indireto } \\
\text { (b) }\end{array}$ & Mediado por & $\begin{array}{c}\text { Efeito } \\
\text { total } \\
(\mathbf{a}+\mathbf{b})\end{array}$ & $\begin{array}{c}\text { Teste t } \\
\text { de } \\
\text { Student }\end{array}$ \\
\hline Imagem & Qualidade & 0,614 & & & 0,614 & 15,685 \\
\hline Qualidade & Satisfação & 0,642 & & & 0,642 & 14,634 \\
\hline Satisfação & Lealdade & 0,696 & & & 0,696 & 16,672 \\
\hline Imagem & Satisfação & & 0,394 & Qualidade & 0,394 & \\
\hline Imagem & Lealdade & & 0,274 & Qualidade/Satisfação & 0,274 & \\
\hline Qualidade & Lealdade & & 0,447 & Satisfação & 0,447 & \\
\hline
\end{tabular}

Fonte: Dados coletados e processados

De acordo com os resultados da Tabela 1, a Imagem exerce efeito direto sobre a qualidade $(0,614)$; esta por sua vez, exerce efeito direto sobre a Satisfação $(0,642)$. E o constructo Satisfação tem efeito direto de 0,696 sobre a lealdade. A Imagem exerce efeito indireto sobre a Satisfação e sobre a Lealdade de 0,394 e 0,274, respectivamente, a primeira sendo mediada pela Qualidade e a segunda mediada pela Qualidade e Satisfação. Finalmente, a Qualidade exerce efeito indireto sobre a Lealdade de 0,447, sendo mediada pela Satisfação.

A partir dos coeficientes estruturais, que produzem efeitos diretos e indiretos, é possível calcular a contribuição dos constructos exógenos para a explicação da variância da Lealdade. 
Os percentuais de contribuição para o percentual da variância explicada para aquele constructo podem ser obtidos associando-se a soma dos coeficientes a $100 \%$. Os valores das parcelas de contribuição podem ser calculados multiplicando-se os percentuais resultantes do cálculo anterior pelo valor da variância explicada, para assim obter fatores de ponderação de cada constructo para essa variância.

Em termos percentuais, a Satisfação explica $49,11 \%$ da variância da Lealdade, a Imagem explica $19,36 \%$ e a Qualidade explica 31,53\% $(49,11 \%+19,36 \%+31,53 \%=100 \%)$. Em termos de parcelas, a contribuição da Satisfação para a variância explicada da Lealdade é de $23,77 \%$, da Imagem é 9,37\% e da qualidade é de 15,26\% $(23,77 \%+9,37 \%+15,26 \%=$ $48,4 \%=\mathrm{R}^{2}$.

A AVE média entre os constructos para o modelo ajustado foi de 0,6245 . O $\mathrm{R}^{2}$ médio calculado foi de 0,4531 , indicando em que grau a variável dependente é prevista pelas independentes. A partir destes valores, calculou-se o índice de adequação do modelo gof $=$ 0,5319 . Tal resultado pode ser aceito como adequado em decorrência de o critério mínimo da AVE ter sido estabelecida em 0,5, conforme Bido et al (2008, p.12).

Na Figura 6, apresentam-se os resultados para as hipóteses relacionadas a este estudo.

Figura 6 - Hipóteses do Modelo

\begin{tabular}{c|lc}
\hline $\begin{array}{c}\text { Número } \\
\text { da } \\
\text { Hipótese }\end{array}$ & \multicolumn{1}{|c}{ Hipótese } & Resultados \\
\hline $\mathrm{H}_{1}$ & A Qualidade é influenciada pela imagem da instituição & Confirmada \\
\hline $\mathrm{H}_{2}$ & A satisfação é influenciada pela qualidade percebida & Confirmada \\
\hline $\mathrm{H}_{3}$ & A Lealdade é influenciada pela qualidade & Não confirmada \\
\hline $\mathrm{H}_{4}$ & A lealdade é influenciada pela satisfação & Confirmada \\
\hline
\end{tabular}

\subsubsection{Testes paramétricos e não-paramétricos para análise das variáveis moderadoras}

A partir dos escores não padronizados resultantes do processamento da técnica de Modelagem de Equações Estruturais, foram realizados testes paramétricos e não-paramétricos para averiguação do papel da variável moderadora curso, sexo e período sobre a influência dos constructos do modelo.

A fim de verificar a possibilidade de efetuar ou não testes paramétricos buscou-se efetuar testes de normalidade. Primeiramente foi aplicado o teste de distribuição normal dos escores não padronizados e verificou-se que apresentam distribuição normal os seguintes constructos: aspectos tangíveis, corpo administrativo, corpo docente, qualidade, imagem e satisfação. Para estes constructos foram aplicados alguns testes paramétricos (ANOVA e $t$ de student). Os constructos que apresentaram distribuição não normal foram: processos administrativos, relação discente, lealdade; neste caso, foram aplicados os testes não paramétricos.

\subsubsection{Testes paramétricos para análise das variáveis moderadoras}

Existe pouca variação entre o grupo sexo e a nota oferecida para as variáveis: aspectos tangíveis, corpo administrativo, corpo docente, qualidade, imagem e satisfação. Este comentário pode ser confirmado com o resultado do teste $t$ de student, na Tabela 2, no qual pelos valores obtidos no Sig, se aceita $\mathrm{H} 0$, ou seja, não há diferença para a variável sexo nas notas dadas para as variáveis pesquisadas. 
Tabela 2- Resultados do teste $t$-student para os constructos

\begin{tabular}{|l|c|c|}
\hline \multicolumn{3}{|c|}{ Independent Samples Test } \\
\hline & $\mathrm{t}$ & Sig. (2-tailed) \\
\hline $\begin{array}{l}\text { ASPECTOS } \\
\text { TANGÍVEIS }\end{array}$ & 0,081 & 0,935 \\
\hline $\begin{array}{l}\text { CORPO } \\
\text { ADMINISTRATIVO }\end{array}$ & $-0,878$ & 0,381 \\
\hline CORPO DOCENTE & $-1,289$ & 0,199 \\
\hline QUALIDADE & $-0,290$ & 0,772 \\
\hline IMAGEM & $-0,265$ & 0,791 \\
\hline SATISFAÇÃO & $-0,231$ & 0,727 \\
\hline
\end{tabular}

Fonte: Dados coletados e processados.

H0: a média é igual nos grupos (masculino e feminino)

Existe pouca variação entre os indivíduos do grupo período e a nota oferecida para as variáveis: aspectos tangíveis, corpo administrativo, corpo docente, qualidade, imagem e satisfação.Nota-se este resultado através do teste t de student (Tabela 3), em que conforme os valores obtidos no Sig, aceita-se H0, ou seja, não existe diferença para a variável período e as notas dadas para as variáveis pesquisadas.

Tabela 3 - Teste $t$-student

\begin{tabular}{|l|c|c|}
\hline \multicolumn{3}{|c|}{ Independent Samples Test } \\
\hline & $\mathrm{T}$ & Sig. (2-tailed) \\
\hline $\begin{array}{l}\text { ASPECTOS } \\
\text { TANGÍVEIS }\end{array}$ & 0,783 & 0,435 \\
\hline $\begin{array}{l}\text { CORPO } \\
\text { ADMINTRATIVO }\end{array}$ & 0,341 & 0,734 \\
\hline $\begin{array}{l}\text { CORPO } \\
\text { DOCENTE }\end{array}$ & & 0,505 \\
\hline QUALIDADE & 0,231 & 0,442 \\
\hline IMAGEM & 0,072 & 0,451 \\
\hline SATISFAÇÃO & 0,488 & 0,481 \\
\hline
\end{tabular}

Fonte: Dados coletados e processados.

Pela ANOVA testou-se $\mathrm{H} 0$, ou seja, a média dos constructos é igual em todos os departamentos. Pelos resultados obtidos na Tabela 4, rejeitou-se H0 para corpo docente e para satisfação ao nível de significância de $5 \%$, pois nestes grupos, encontra-se pelo menos uma comparação em que a média é diferente de zero. Portanto, com exceção de corpo docente e satisfação, os departamentos foram igualmente avaliados nos outros quesitos.

Tabela 4- Resultados do Teste $\mathbf{F}$ para a variável curso

\begin{tabular}{|l|c|c|c|c|c|}
\hline & umo f Squares & df & Mean Square & F & Sig. \\
\hline ASP_TANG & 6,27 & 3 & 2,09 & 1,082 & 0,358 \\
\hline C_ADMINT & 9,416 & 3 & 3,139 & 1,354 & 0,258 \\
\hline C_DOCENT & 56,831 & 3 & 18,944 & 9,452 & 0,0 \\
\hline QUALID & 5,244 & 3 & 1,748 & 1,95 & 0,122 \\
\hline IMAGEM & 7,333 & 3 & 2,444 & 1,443 & 0,231 \\
\hline SATISFAÇ & 21,054 & 3 & 7,018 & 4,338 & 0,005 \\
\hline
\end{tabular}

A análise de variância por si só permite identificar que existe diferença significativa entre as médias, mas não permite identificar quais médias de quais departamentos são 
significativamente diferentes. A partir de uma análise de contrastes, pelo teste de Tukey, é possível identificar quais médias são estatisticamente diferentes, resultados apresentados na Figura 7.

Figura 7 - Resultados dos testes paramétricos para a variável curso

\begin{tabular}{|l|l|}
\hline $\begin{array}{l}\text { ASPECTOS } \\
\text { TANGÍVEIS }\end{array}$ & $\begin{array}{l}\text { Não existe diferença entre a opinião dos alunos dos quatro cursos quanto } \\
\text { aos aspectos tangíveis. }\end{array}$ \\
\hline $\begin{array}{l}\text { CORPO } \\
\text { ADMINISTRATIVO }\end{array}$ & $\begin{array}{l}\text { Não existe diferença entre a opinião dos alunos dos quatro cursos quanto ao } \\
\text { corpo administrativos. }\end{array}$ \\
\hline CORPO DOCENTE & $\begin{array}{l}\text { Existe diferença entre a opinião dos alunos dos quatro cursos quanto ao } \\
\text { corpo docente, sendo que os alunos do curso de Atuária fazem uma } \\
\text { avaliação superior em relação à avaliação feita pelos alunos dos outros } \\
\text { cursos, vindo a seguir a avaliação feita pelos alunos de Ciências Contábeis. }\end{array}$ \\
\hline QUALIDADE & $\begin{array}{l}\text { Não existe diferença entre a opinião dos alunos dos quatro cursos quanto à } \\
\text { qualidade do curso. }\end{array}$ \\
\hline IMAGEM & $\begin{array}{l}\text { Não existe diferença entre a opinião dos alunos dos quatro cursos quanto à } \\
\text { imagem. }\end{array}$ \\
\hline SATISFAÇÃO & $\begin{array}{l}\text { Existe diferença entre a opinião dos alunos dos quatro cursos quanto à } \\
\text { satisfação, sendo que os alunos do curso de Atuária fazem uma avaliação } \\
\text { superior em relação à avaliação feita pelos alunos dos outros cursos. }\end{array}$ \\
\hline
\end{tabular}

Fonte: elaborado pelo autor

\subsubsection{Testes não paramétricos para análise das variáveis moderadoras}

Foram realizados os testes não paramétricos a partir dos escores resultantes do processamento da técnica Modelagem de Equações Estruturais, para averiguação do papel das variáveis moderadoras sexo, curso e período, na influência sobre os constructos relação discente, lealdade e processos administrativos. Aplicando-se o teste Kruskal-Wallis para a variável moderadora curso, com quatro categorias (Administração, Contábeis, Economia e Atuária), rejeita-se a hipótese de igualdade de médias para os constructos relação discente e lealdade.

Tabela 5- Resultado do teste Kruskal-Wallis

\begin{tabular}{|l|c|c|c|}
\hline & $\begin{array}{c}\text { PROCESSOS } \\
\text { ADMINISTRATIVOS }\end{array}$ & $\begin{array}{c}\text { RELAÇÃO } \\
\text { DISCENTE }\end{array}$ & LEALDADE \\
\hline Chi-Square & 7,507 & 13,44 & 14,727 \\
\hline df & 3 & 3 & 3 \\
\hline Asymp. Sig. & 0,057 & 0,004 & 0,002 \\
\hline
\end{tabular}

Fonte: Dados coletados e processados.

Aplicando-se o teste Mann-Whitney para a variável moderadora sexo e período, não se rejeita a hipótese de que a amostra possui médias iguais, nos estratos das variáveis sexo e período para os constructos processos administrativos, relação discente e lealdade, ao nível de significância de 5\%. Assim, estes resultados sugerem que as variáveis sexo e período não exercem influência sobre os constructos considerados para esta análise (Tabelas 6 e 7).

Tabela 6 - Resultados do Teste Mann-Whitney para a variável sexo

\begin{tabular}{|l|r|r|r|}
\hline Test Statistics & \multicolumn{3}{|c|}{ Grupo de Variável: Sexo } \\
\hline & PRO_ADM & R_DISCEN & LEALDADE \\
\hline Mann-Whitney U & 5787,5 & 5437 & 5945 \\
\hline Wilcoxon W & 15517,5 & 15167 & 9773 \\
\hline Z & $-0,541787446$ & $-1,274355769$ & $-0,21222055$ \\
\hline Asymp. Sig. (2-tailed) & 0,587964952 & 0,202537358 & 0,831934988 \\
\hline
\end{tabular}

Fonte: Dados coletados e processados. 
Tabela 7 - Resultado do teste Mann-Whitney para a variável período

\begin{tabular}{|l|r|r|r|}
\hline Test Statistics & \multicolumn{3}{|c|}{ Grupo de Variável: Período } \\
\hline & PRO_ADM & \multicolumn{1}{|c|}{ R_DISCEN } & LEALDADE \\
\hline Mann-Whitney U & 5774,5 & 5853,5 & 6122 \\
\hline Wilcoxon W & 11027,5 & 13603,5 & 11375 \\
\hline Z & $-1,123965502$ & $-0,961906195$ & $-0,41297984$ \\
\hline Asymp. Sig. (2-tailed) & 0,261027664 & 0,336096734 & 0,679621398 \\
\hline
\end{tabular}

Fonte: Dados coletados e processados.

Assim, pode-se resumir o resultado dos teste não-paramétricos na Figura 8.

Figura 8 - Testes não-paramétricos para a variável curso

\begin{tabular}{|l|l|}
\hline PROCESSOS & $\begin{array}{l}\text { Não existe diferença entre a opinião dos alunos dos quatro cursos quanto } \\
\text { aDMINISTRATIVOS }\end{array}$ \\
\hline RELAÇAO & $\begin{array}{l}\text { Existe diferença entre a opinião dos alunos dos quatro cursos, sendo que os } \\
\text { alunos de atuária e administração fazem uma avaliação superior. }\end{array}$ \\
\hline DISCENTE & $\begin{array}{l}\text { Existe diferença entre a opinião dos alunos dos quatro cursos, sendo que os } \\
\text { alunos de atuária e ciências contábeis são mais leais. }\end{array}$ \\
\hline
\end{tabular}

Fonte: elaborado pelo autor

\section{CONSIDERAÇÕES FINAIS}

Este estudo teve como questão-problema a seguinte indagação: "Quais são os fatores que contribuem para a lealdade do aluno para que este permaneça e termine o curso superior escolhido?". Para responder a este questionamento, foi definido o objetivo de identificar os fatores que contribuem para que o aluno fique satisfeito e seja leal ao curso escolhido.

O modelo escolhido para responder a questão-problema foi o proposto por Bloemer (1998) e aplicado anteriormente para instituições financeiras. Neste estudo o modelo foi aplicado em uma instituição de ensino superior. Foi feita uma adaptação ao modelo de Bloemer (1998), com constructos e variáveis que contemplam os quesitos para avaliar uma instituição de ensino, com base em vários autores citados ao longo do trabalho, como qualidade, imagem, satisfação e lealdade.

Para buscar a correlação destes constructos (qualidade, imagem, satisfação e lealdade), foi escolhida a técnica de equações estruturais por ser uma técnica multivariada que combina aspectos de regressão múltipla e análise fatorial para estimar uma série de relações de dependência inter-relacionadas simultaneamente, atendendo as exigências desta pesquisa. $O$ constructo qualidade foi segmentado em cinco blocos, contendo questionamentos sobre: corpo docente, corpo técnico-administrativo, relação discente, aspectos tangíveis, processos administrativos. O modelo utilizado como referência foi o de Bloemer (1998), buscando-se confirmar as seguintes hipóteses do trabalho:

- $\mathrm{H}_{1}$ : A Qualidade é influenciada pela imagem da instituição;

- $\mathrm{H}_{2:}$ A satisfação é influenciada pela qualidade percebida;

- $\mathrm{H}_{3}$ : A Lealdade é influenciada pela qualidade;

- $\mathrm{H}_{4}$ : A lealdade é influenciada pela satisfação.

Aplicando-se a técnica de equações estruturais na base de dados da pesquisa, ou seja, os alunos matriculados nos quatro cursos (Administração, Ciências Contábeis, Ciências Atuariais e Economia) da FEA/USP que responderam a pesquisa, pode-se notar que apenas as hipóteses 1,2 e 4 foram confirmadas.

A partir dos escores não padronizados resultantes do processamento da técnica de Modelagem de Equações Estruturais, foram realizados testes paramétricos e não-paramétricos para averiguação do papel da variável moderadora curso, sexo e período sobre a influência dos 
constructos do modelo. Pode-se notar que existe pouca variação entre a variável sexo e as notas obtidas para os constructos que compõem a qualidade, imagem e satisfação, bem como os itens que compõem a qualidade (corpo docente, corpo técnico-administrativo, aspectos tangíveis, processos administrativos). O mesmo ocorreu com a variável período. Pode-se dizer que as variáveis sexo dos alunos e período em que os alunos estudam na instituição mostram pouca variação nas notas oferecidas para os constructos da pesquisa.

Com base no teste Anova, nota-se, de maneira geral, uma avaliação por parte dos alunos de Contábeis e Atuária, superior à avaliação que fazem os alunos de Administração e de Economia de seu corpo docente. No entanto, com relação à satisfação, as diferenças são menos sensíveis, existindo uma diferença estatisticamente significativa apenas entre as respostas dos alunos de Ciências Contábeis e as respostas dos alunos de Administração, sendo que as respostas dos alunos de Ciências Contábeis têm média estatisticamente superior à média das respostas dos alunos de Administração, para a variável satisfação.

Todo trabalho de pesquisa possui limitações e/ou restrições que devem ser reveladas. Este estudo apresenta restrições de ordem teórica e metodológica.

De ordem teórica, este estudo utilizou-se do modelo de Bloemer (1998)) e SERVPERF de Cronin e Taylor $(1992,1994)$ aplicado ao setor de ensino superior. Assim, pode-se considerar que outras variáveis podem ter sido desconsideradas no desenvolvimento do modelo deste trabalho. Quanto às limitações de ordem metodológica, a amostra utilizada é de caráter não probabilístico, determinada pela dinâmica dos respondentes na internet, veículo este utilizado para coletar os dados da pesquisa.

Cabe ressaltar que o resultado desta pesquisa não deve ser extrapolado para outro universo, pois este estudo limitou-se a uma única instituição de ensino superior.

Um estudo comparativo entre uma instituição pública e uma instituição privada poderia ser realizado para se compararem as relações descobertas neste trabalho entre os constructos qualidade, imagem, satisfação e lealdade.

\section{REFERÊNCIAS BIBLIOGRÁFICAS}

AAKER, David A. et.al. Pesquisa de Marketing. 2. ed. São Paulo: Atlas,2004.

BAPTISTA, P. P. Lealdade do consumidor e os seus antecedentes: um estudo aplicado ao setor varejista na internet. São Paulo, 2005. Tese (Doutorado em Administração). Programa de Pós-Graduação em Administração, Faculdade de Economia, Administração e Contabilidade da Universidade de São Paulo.

BIDO, D. S.; GODOY, A. S.; FERREIRA, J. F. KENSKI, J. M.; SCARTEZINI, V. N. Examinado a relação entre aprendizagem individual, grupa e organizacional em uma instituição financeira. In: ENCONTRO DA ASSOCIAÇÃO NACIONAL DE PÓSGRADUAÇÃO E PESQUISA EM ADMINISTRAÇÃO - ENANPAD 32, 2008. Rio de Janeiro, anais eletrônicos. Rio de Janeiro: ANPAD, 2008. 1 CD-ROM.

BLOEMER, J. RUYTER, K. PEETERS, P. Investigating drivers of bank loyalty: the complex relationship between image, service quality and satisfaction. Internacional Journal of Bank Marketing. Bradford: MCB University Press, v.16, n.7, p. 276-286, 1998.

BRYMAN, A. Research methods and organization studies. London. Uniwin Hyman, 1989.

CRONIN JR., J.Joseph: TAYLOR, Steven A. Measuring service quality: a reexamination and extension. Journal of Marketing. Chicago, v.56, n. 9. p. 55-68, 07/1992.

DIAS, E. T. Dal Mas. 1995. Dúvida da continuidade dos estudos universitários: uma questão adolescente. Dissertação de Mestrado. IP/USP São Paulo. 247 p.

DIAS, M.; ZWICKER, R.; VICENTIN, I. A evolução do Modelo de Aceitação de Tecnologia de Davis: uma análise do original e variações de 1985 até 2003. In: ASAMBLEA ANUAL DEL CONSEJO LATINOMAEIRCANO DE ESCUELAS DE 
ADMINISTRACIÓN - CLADEA. Lima: CLADEA/Universidad San Ignacio de Loyola, 2003.

DICK, Alan S.;BASU, Kunal. Customer Loyalty: toward an integrated conceptual framework. Journal of academy of marketing science. $v$ 22, n.2,p.99-113.

FARIAS, S.; SANTOS, R. Modelagem de equações estruturais e satisfação do consumidor: uma investigação teórica e prática. RAC - Revistas de Administração Contemporânea, vol. 4, n. 3, p. 107-132, set./dez. 2000.

FITZSIMMONS, James A; FITZSIMMONS, Mona J. Administração de Serviços: operações, estratégia e tecnologia da informação. 4.ed. Porto Alegre: Bookman, 2005.

FORNELL, Claes. A national consumer satisfaction barometer: the Swedish experience. Journal of Marketing. Chicago, v. 56, p. 6-21, 01/1992.

FORNELL, Claes et al. The american customer satisfaction index: natures, purpose, and findings. Journal of Marketing. Chicago, v. 60, p. 7-18, 10/1996.

GRÖROOS, C.. Marketing: gerenciamento e serviços: a competição por serviços na hora da verdade. Rio de Janeiro: Campus, 1993.

GUIMARAES, R.T. Avaliação de marca das instituições de ensino superior no segmento de mestrado e doutorado em administração: uma abordagem multivarida. Doutorado. FEA/USP,2005.

INSTITUTO BRASILEIRO DE GEOGRAFIA E ESTATISTICA - IBGE. Homepage Institucional, 2005. Pesquisa Anual de Serviços. Rio de Janeiro, vol. 7, p.1-196, 2005. Disponível em: http://www.ibge.gov.br Acesso em: julho, 2007.

KOTLER, Philip .Administração de marketing: a edição do novo milênio. São Paulo: Pratice Hall, 2000.

Administração de marketing, Rio de Janeiro: Atlas, 1998.

. FOX, Karen. Marketing estratégico para instituições educacionais. São Paulo: Atlas, 1994.

. Marketing management. 8 ed. New-Jersey: Pretice-Hall, 1994.

KOTLER, P; ANDREASEN, A. R. Strategic marketing for nonprofit organizations. 5 ed. Pretice Hall, 1996.

KOTLER, P; ARMSTRONG, Gary. Princípios de Marketing. 5 ed. Rio de Janeiro: Prentice Hall, 1993. 478 p.

MALHOTRA, N. Pesquisa de Marketing: uma orientação aplicada. 4 ed. Porto Alegre: Bookman, 2006.

MANES, J. M.. Marketing para instituciones educativas: guía para planificar la captación y retención de alumnos. Buenos Aires: Granica, 1997.

MASANO, A. C. R. Expectativas e Percepções do Mercado Bancário de Pessoas Físicas de Alta Renda no Município de São Paulo. Dissertação de Mestrado apresentada ao programa da FEA/USP, São Paulo, 2006.

MOISES FILHO, J.G. Qualidade do ensino e eficiência técnica no ensino superior privado: o caso do distrito federal. Dissertação apresentada a Universidade de Brasília, $2006 . \quad$ http://bdtd.bce.unb.br/tedesimplificado/tde_arquivos/61/TDE-2007-0201T161341Z-636/Publico/Dissert_Jorge\%20Gabriel.pdf <acesso em 10/10/2007>

NEAL, W. D. Satisfaction is Nice, but value drives loyalty. Marketing Research. v. 11, p.20-23, spring 1999.

OLSEN,S. O. Comparative evaluation and the relationship between quality, satisfaction, and repurchase loyalty. Journal of the Academy of Marketing Science. V.30, n. 3, p. 240249, 2002.

PARASURAMAN, A. ZEITHAML, V.; BERRY, L. A conceptual model of services quality and its implications for future research. Journal of Marketing. Chicago, v. 49, p. 41-50, Fall 1985. 
A multiple-item scale for measuring consumer perceptions. Journal of Retailling. New York: New York University Press, v. 64, p. 12-29, Spring 1988.

Reassessment of expectations as a comparison standard in measuring service quality: implications for further research. Journal of Marketing. Chicago, v. 58, n. 1, p. 111-124, 01/1994.

PERFEITO, J.; BECKER, M.; SILVEIRA, A.; FORNONI, M. http://www.inpeau.ufsc.br/coloquio04/completos/JuarezPerfeito\%20\%20MARKETING\%20EM\%20INSTITUI\%C7\%D5ES.doc <acesso 23 de outubro de 2007.

SELNES, F. An examination of effect of product performance on brand reputation, satisfaction and loyalty. The Journal of Product and brand management, Santa Barbara, v.2, p. 45-60, 1993.

ZEITHAML, V.; PARASURAMAN, A.; BERRY, L. A conceptual model of service quality and its implications for future research. Journal of Marketing, vol. 49, n. 4, p. 41-50, 1985.

. Delivering quality service: balancing customer perceptions and expectations. New York: the Free Press, 1990.

- The behavioral consequences of service quality. Journal of Marketing. Chicago, v. 60, n. 2, p. 31-46, 04/1996.

ZEITHAML, V. A., BITNER, M. J. Marketing de serviços: a empresa com foco no cliente. 2 ed. Porto Alegre: Bookaman, 2003.

YIN, R. K. Case Study research - design and methods. London, sage, 1989.

ZWICKER, R.; SOUZA, C. A.; BIDO, D.S. Uma revisão do modelo do grau de informatização de empresas: novas propostas de estimação e modelagem usando PLS (partial least squares). In: ENCONTRO DA ASSOCIA̧ÇÃO NACIONAL DE PÓSGRADUAÇÃO E PESQUISA EM ADMINISTRAÇÃO - ENANPAD 32, 2008. Rio de Janeiro, anais eletrônicos. Rio de Janeiro: ANPAD, 2008. 1 CD-ROM. 